\title{
Los suelos del área de influencia del Códice Santa María Asunción y su representación pictórica
}

\author{
Soils of the area of influence of Santa María Asunción Codex and the pictorial representation
}

\author{
Ma. del Carmen Gutiérrez Castorena ${ }^{1 *}$, Carlos Alberto Ortiz Solorio ${ }^{1}$, \\ Beatriz Stephanie Fernández Galán ${ }^{1}$, Edgar Vladimir Gutiérrez Castorena ${ }^{2}$ y Tania González Vargas ${ }^{1}$ \\ ${ }^{1}$ Programa de Edafología, Colegio de Postgraduados, Campus Montecillo. Km 36.5 Carretera México-Texcoco. 56230 Texcoco, Estado de México, México. \\ Autora responsable (castor@colpos.mx) \\ ${ }^{2}$ Facultad de Agronomía. Universidad Autónoma de Nuevo León. Francisco Villa s/n, Ex hacienda "El Canadá”. 66050 Gral. Escobedo, N. L., México.
}

\section{RESUMEN}

A pesar de que el Códice de Santa María Asunción (CSMA) ha sido ampliamente estudiado, el levantamiento de suelos en el cual se sustentó para interpretar los glifos de diferentes clases de tierras, fue realizado fuera del área de influencia del códice. Reinterpretar los glifos de clases de tierras a través de un estudio de suelos, geomorfología y materiales parentales es el objetivo de la presente investigación. Cinco barrios que aparecen en el CSMA fueron estudiados y se cuantificó la frecuencia de los glifos en cada uno de ellos. Posteriormente, se realizaron recorridos de campo parcela por parcela y se colectaron muestras de suelos y tobas (tepetates) para su análisis en el laboratorio y clasificación taxonómica. Los resultados indicaron que el área de influencia del CSMA está integrada por Regosols/Leptosols (formados a partir de riolita, andesita y tobas), Anthrosols (terrazas) y Fluvisols. Por la frecuencia de los glifos y el tipo de suelo se puede indicar que Tepetatlalli (tepetates) puede representar a las terrazas, Xalallis a agregados granulares $(<3 \mathrm{~mm})$ y suaves y no suelos arenosos; el Tezoquilt a los bloques subangulares/granulares, duros o suaves $(<2 \mathrm{~cm})$ o tierra; Tlalcoztli a las tierras amarillas polvosas y de baja calidad agrícola; en tanto, Atoctlis y Tezoquitl/Xalalli a los suelos aluviales activos y pasivos. Respecto a lo anterior se concluyó que los nahuas clasificaban a las tierras con base en el tamaño de los agregados, consistencia del suelo y origen (natural y artificial) y no por textura ni por color.

Palabras clave: Etnoedafología, clasificación de suelos, clases de tierras, Anthrosols.

Cita recomendada:

Gutiérrez Castorena, M. C., C. A. Ortiz Solorio, B. E. Fernández Galán, E. V. Gutiérrez Castorena y T. González Vargas. 2017. Los suelos del área de influencia del Códice Santa María Asunción y su representación pictórica. Terra Latinoamericana 35: 101-111.

\section{SUMMARY}

Although the Santa Maria Asunción Codex (SMAC) has been extensively studied, the soil survey on which it was based to interpret the glyphs of different kinds of land was performed outside the area of influence of the codex. Reinterpreting the glyphs of different land classes through a study of soils, geomorphology and parent materials is the objective of this research. Five neighborhoods that appear in the SMAC were studied, and the frequency of the glyphs in each one of them was quantified. Subsequently, plot-by-plot field visits were carried out and samples of soil and tuffs (tepetates) were collected for laboratory analysis and taxonomic classification. The results indicate that the area of influence of the SMAC is composed of Leptosols/Regosols (formed from rhyolite, andesite and tuffs), Anthrosols (terraces) and Fluvisols. The frequency of glyphs and type of soil indicated that Tepetatlalli (tepetates) can represent terraces, Xalallis granular peds $(<3 \mathrm{~mm})$ and non-sandy soils; Tezoquilt subangular blocky/granular peds with hard and soft consistency $(<2 \mathrm{~cm})$; Tlalcoztli dusty yellow land of low agricultural quality; and Atoctlis and Tezoquitl/ Xalalli correspond to active and passive alluvial soils, respectively. The conclusion is that the Nahua classified their lands based on aggregate size, soil consistency, and origin (natural and artificial), and not on texture or color.

Index words: Ethnoedaphology, soil classification, land classes, Anthrosols. 


\section{INTRODUCCIÓN}

El Códice de Santa María Asunción (CSMA) es un manuscrito de ochenta folios que se elaboró en el periodo de contacto entre los nativos y los europeos (alrededor de 1546) en Tepetlaoxtoc, Edo. de México y ha sido objeto de una intensa investigación, específicamente relacionada con los censos de población, parcelas (perímetros y superficies), tipos de suelos (taxonomía popular) y su aritmética (Harvey y Williams, 1980; Williams y Ortiz-Solorio, 1981; Williams 1989, 1992, 2006; Williams y Jorge y Jorge, 2008; López-Corral, 2011; Williams y Pierce, 2014).

El titulo original del CSMA es Apeo y Deslinde de las tierras de Santa María Asunción, es un códice que contiene notación pictográfica Nahua (específicamente de la división tribal acolhua), y está dividido en 12 barrios (Williams, 2006). Cada barrio está subdividido a su vez en tres partes: Tlacatlacuilolli (censo de población a nivel familiar), Milcocoli (listado de parcelas con datos perimetrales) y Tlahuelmantli (parcelas con información de sus áreas), asociadas con jefes de familias.

Las parcelas tienen uno o varios glifos que representan clases de tierra, las cuales se clasificaron en términos de textura, color y calidad agrícola (Williams y Ortiz-Solorio, 1981; Williams, 2006; Williams y Pierce, 2014). Con base en esta clasificación, en el CSMA se representaron siete clases de tierras: Tlalcoztli (tierra amarilla); Tezoquitl (tierra negra de barro); Xalalli (tierra arenosa); Atoctli (tierras formadas por el acarreo de partículas por el agua); Tlaixtli (tierras de laderas); Tepetlatlalli (tierras recuperadas o tepetates) y Tetlalli (tierras pedregosas). En cuanto a calidad agrícola, Tepetlatlalli son las de menor calidad (Williams, 1972; 1992); mientras que las Tlalcoztli y las Tezoquitl son las más productivas (Sahagún, 1963; Williams y Pierce, 2014). Asimismo, cada clase de tierra puede tener entre dos o más variantes: por ejemplo, Tlalcoztlis (35), Tepetlatlalli (28), Tezoquitl(11), A toctli (11) de acuerdo con el CSMA. El ambiente volcánico con diversidad de estructuras geológicas (GEM, 1993); además de sedimentos coluviales y aluviales y pequeños domos volcánicos (Williams y Pierce, 2014), pueden ser los factores responsables de esta gran variedad de clases de tierras.

El problema de las investigaciones antes citadas es que la interpretación de los glifos no se basó en los suelos donde los acolhuas representaron a las clases de tierras. Williams y Ortiz-Solorio (1981), autores que son referencia para el estudio de suelos, describieron perfiles y utilizaron un levantamiento de series de suelos del área de influencia de Chapingo (Cachón et al., 1976), que se llevó a cabo fuera del área de influencia del CSMA. En consecuencia, la interpretación de los glifos con estudios detallados de suelos y del ambiente en cada uno de los barrios reportados en el CSMA, podría desglosar nueva información que ayudaría a comprender mejor la avanzada clasificación y el sistema complejo de notación de tipos de tierras que los nahuas del centro de México representaban mediante el uso de glifos (Williams, 1980; 1989; 2006). Las interrogantes que se tuvieron en el presente estudio fueron: ¿hay tanta variedad de suelos y materiales parentales en el área de influencia del CSMA?, ¿Cuáles son las propiedades del suelo que fueron representadas en los glifos?

El objetivo de la presente investigación fue reinterpretar los glifos de clases de tierras representados en el CSMA a través de su cuantificación en cada barrio, y un levantamiento de suelos detallado, de materiales parentales y geomorfología.

\section{MATERIALES Y MÉTODOS}

\section{Área de Estudio}

El área de influencia del Códice de Santa María Asunción (CSMA) cubre una superficie de 256 ha, con base en el mapa de Williams (1991) y está incrustado en el municipio de Tepetlaoxtoc (náhuatl Tepetl = tepetate, y Ozto: cueva o gruta), Estado de México. Este municipio se localiza en el extremo oriente del valle de México, entre las coordenadas $19^{\circ} 38^{\prime} 44^{\prime \prime}$ y $19^{\circ}$ 27' 43 de N, 98 39' 10 y $98^{\circ} 52^{\prime}$ ' $10^{\prime \prime}$ 'O y una altitud entre 2300 y 3000 m (Figura 1). La región presenta diversidad de estructuras geológicas, básicamente del proceso volcánico-tectónico iniciado a finales del Paleozoico y concluido durante el Mesozoico (GEM, 1993), lo que resulta en paisajes de lomeríos y planos aluviales. El clima es BS (c) wk'g (seco estepario), de acuerdo con la clasificación de Köppen, modificado por García (1973). Los suelos fueron clasificados como dominantes son Feozems y Vertisols, en donde se cultivan principalmente maíz bajo agricultura de secano (GEM, 1993). 

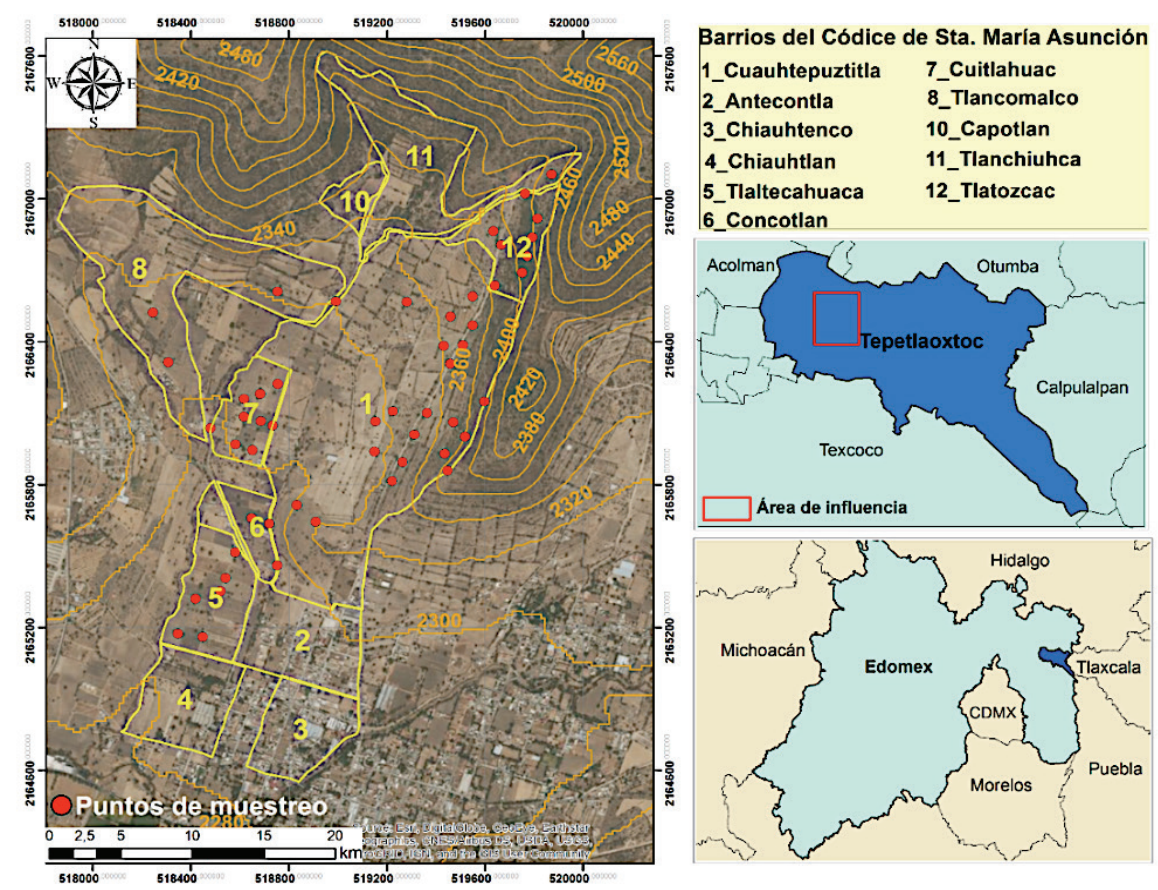

Figura 1. Localización de los sitios de muestreo en los barrios del CSMA (a partir de Williams, 1991).

\section{Identificación Espacial de las Clases de Tierras}

Una copia digital del CSMA fue proporcionada por la Biblioteca Nacional de México. Los barrios que se analizaron en este estudio corresponden a la fracción del Milcocoli, en donde se representan los perímetros de las parcelas. Cada glifo de clase de tierra y sus variantes fueron cuantificados para determinar su frecuencia en cada uno de los barrios. Posteriormente, el mapa realizado por Williams (1991) fue utilizado para ubicar y digitalizar los 12 barrios a través del mapa base de ArcGis 10.3 como se reporta en la Figura 1. Finalmente, se realizaron mosaicos de imágenes directamente del google earth para ampliar la resolución y elaborar un mapa base de cada barrio.

\section{Recorridos de Campo}

Los barrios que se analizaron fueron: Cuauhtepuztitla (Barrio 1), Tlaltecahuaca (Barrio 5), Concotlan (Barrio 6), Cuitlahuac (Barrio 7), Tlancomalco (Barrio 8) y Tlantoztoc (Barrio 12). Los otros Barrios (2, 3, 4, 10, y 11) fueron excluidos de la presente investigación por su urbanización, parcelas cercadas y el Barrio 9 que corresponde al Códice Vergara. La representación pictórica y nombre de los diferentes glifos de clases de tierras se reportan en la Figura 2.
En cada barrio analizado se llevó a cabo un recorrido de campo, parcela por parcela, utilizando el mapa base, con el fin de registrar construcciones prehispánicas (terrazas), geomorfología (mesetas, pendientes y valles), pedregosidad y materiales parentales (rocas y tobas). Asimismo, se llevaron a cabo barrenaciones (una o dos por parcela) para registrar color, profundidad y consistencia del suelo. Cuando se presentaron cambios en estas variables se colectaron muestras de suelo (aproximadamente $2 \mathrm{~kg}$ ) a diferentes profundidades $(0-20 \mathrm{~cm}$ y $20-40 \mathrm{~cm})$, dando un total de 54 puntos de muestreo (Figura 1). Además, se tomaron muestras de tepetate o materiales endurecidos, para su caracterización mineralógica.

Las propiedades del suelo se determinaron tomando como referencia al manual de procedimientos analíticos de Van Reeuwijk (ISRIC, 2003). Los análisis efectuados fueron: $\mathrm{pH}$ relación agua-suelo 1:1; materia orgánica (MO) por el método de Walkley y Black; color en seco y húmedo empleando la carta de colores Munsell; densidad aparente (Dap), por el método de la parafina y distribución del tamaño de partícula (método de la pipeta). Además, se analizó la estructura directamente en campo (tipos, tamaños y consistencia). Los suelos se clasificaron utilizando IUSS Grupo de Trabajo WRB (2015).

La mineralogía de la fracción gruesa de las tobas volcánicas (tepetates) se determinó por conteo de 


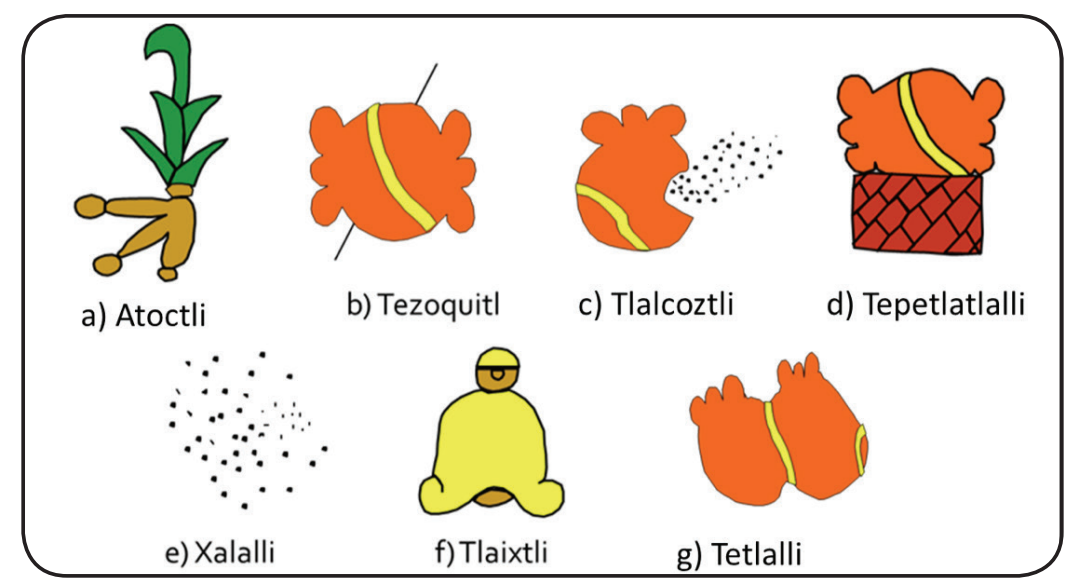

Figura 2. Glifos de las diferentes clases de tierras del CSMA: a) tierras formadas por el acarreo de partículas por el agua; b) tierra negra de barro; c) tierra amarilla; d) tierras recuperadas o tepetates; e) tierra arenosa; f) tierras en las laderas; g) tierras pedregosas (a partir de Williams, 1981).

puntos bajo un microscopio petrográfico utilizando la metodología de Ingersoll et al. (1984). Las tobas se clasificaron con base en Williams et al. (1968).

\section{RESULTADOS Y DISCUSIÓN}

\section{Tipos de Suelos y Materiales Parentales}

En general, en el área de estudio ocurren Leptosols/ Regosols por la profundidad del suelo, Anthrosols, por la construcción de terrazas y Fluvisols en el plano aluvial, los cuales se distribuyen en catenas. En la zona no hay Vertisols o Feozems como se reportó en GEM (1993). En la Figura 3 se ilustra la catena del Barrio 1 que cubre mayor extensión en el CSMA, mayor número de parcelas y tipo de suelos. Además, en el Cuadro 1 se reportan las principales propiedades edáficas de los horizontes superficiales de 17 puntos representativos de clases de tierras de los barrios estudiados.

A continuación, se describen los tipos de suelos y su ocurrencia en el paisaje.

a) Eutric Leptosols/ Eutric Regosols (Leptic) o suelos residuales. Estos suelos se formaron a partir de riolita (Barrios 1 y 12) o de andesita (Barrio 12); con una

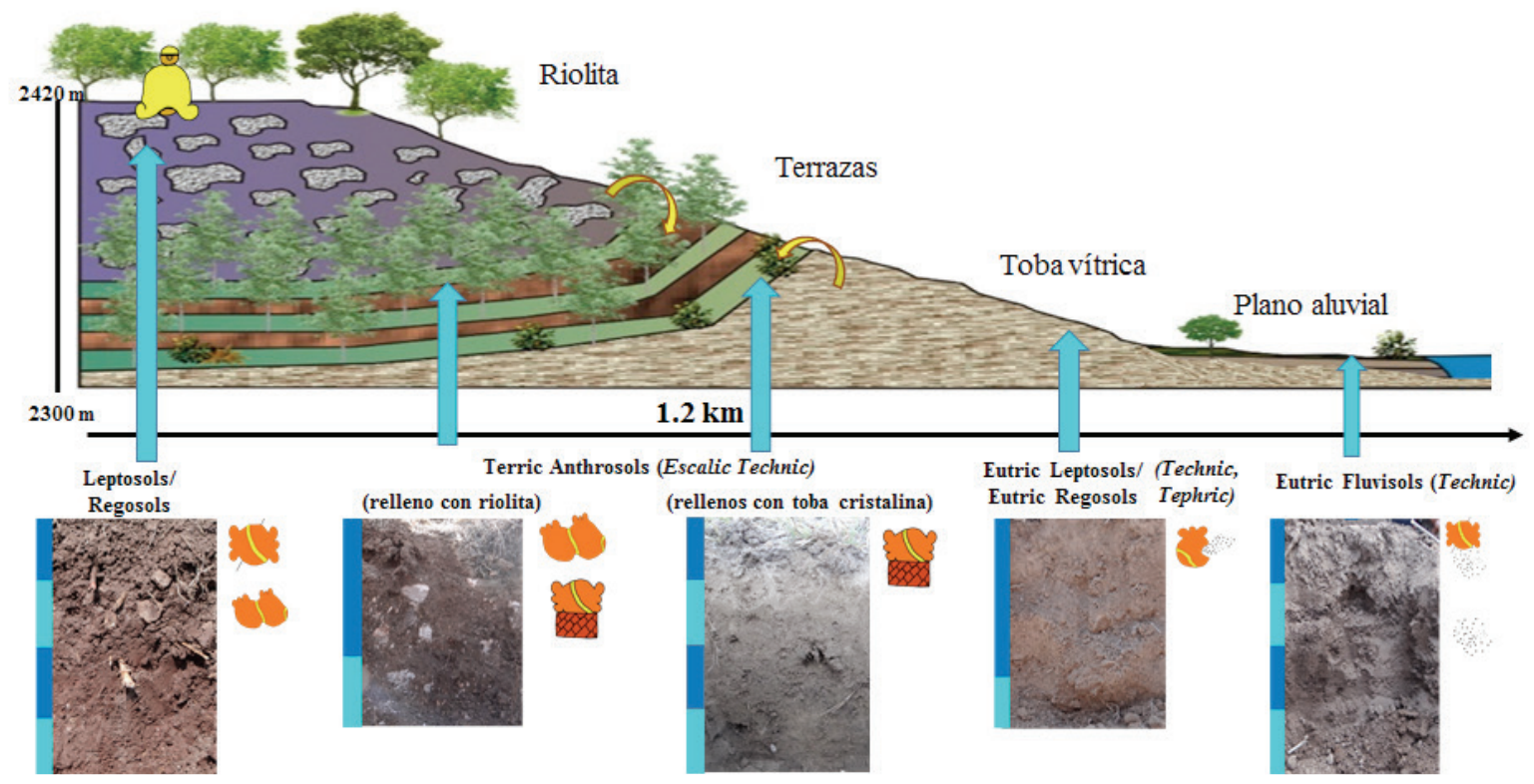

Figura 3. Catena de suelos del Barrio 1-Cuahtepuztitla. 
Cuadro 1. Propiedades físicas y químicas de los suelos de los barrios estudiados.

\begin{tabular}{|c|c|c|c|c|c|c|c|c|}
\hline \multirow{2}{*}{ Barrio } & \multirow{2}{*}{ Profundidad } & \multirow{2}{*}{ Estructura } & \multicolumn{2}{|c|}{ Color } & \multirow{2}{*}{ Dap } & \multirow{2}{*}{$\mathrm{pH}$} & \multirow{2}{*}{ MO } & \multirow{2}{*}{ Clase textural } \\
\hline & & & Seco & Húmedo & & & & \\
\hline & $\mathrm{cm}$ & & & & $\mathrm{g} \mathrm{cm}^{-3}$ & & $\%$ & \\
\hline \multicolumn{9}{|c|}{ Eutric Leptosols/ Eutric Regosols (Leptic) a partir de roca riolítica/andesita } \\
\hline 1 & $0-20$ & Subangular & $10 \mathrm{YR} 3 / 2$ & $10 \mathrm{YR} 4 / 2$ & 1.6 & 6.8 & 2.5 & Arcillosa \\
\hline 1 & $0-20$ & Angular & 10YR 6/3 & 10YR 3/1 & 1.4 & 7.6 & 2.4 & Franco arcillosa \\
\hline \multirow[t]{2}{*}{12} & $0-20$ & Granular & 7.5 YR $6 / 2$ & $2.5 \mathrm{YR} 4 / 2$ & 1.5 & 7.4 & 1.23 & Franco arcillosa \\
\hline & $20-40$ & Subangular & $5 Y R 7 / 1$ & $10 \mathrm{YR} 6 / 2$ & 1.7 & 7.8 & 0.96 & Franco arcillosa \\
\hline 12 & $0-20$ & Granular y subangular & $10 Y R 5 / 4$ & 7.5 YR 3/2 & 1.5 & 7.9 & 1.1 & Franco arcillosa \\
\hline \multicolumn{9}{|c|}{ Terric Anthrosols (Escalic. Technic) con rellenos de roca riolítica (terrazas) } \\
\hline \multirow[t]{2}{*}{1} & $0-10$ & Subangular & $10 Y R 3 / 2$ & 10YR 2/1 & 1.7 & 6.2 & 4.2 & Franco arenosa \\
\hline & $10-40$ & Subangular & $10 \mathrm{YR} 3 / 2$ & $10 \mathrm{YR} 2 / 2$ & 1.2 & 6.1 & 3.41 & Arcillosa \\
\hline 1 & $0-40$ & Subangular & 10YR $3 / 2$ & $10 \mathrm{YR} 4 / 2$ & 1.6 & 6.8 & 3.1 & Arcillosa \\
\hline \multirow[t]{2}{*}{12} & $0-22$ & Granular y subangular & $10 \mathrm{YR} 5 / 2$ & 10YR 3/1 & 1.5 & 6.5 & 2.3 & Franco arcillo limosa \\
\hline & $22-40$ & Subangular & $10 \mathrm{YR} 4 / 2$ & 10YR 3/1 & 1.6 & 6.7 & 2.34 & Franco arcillo limosa \\
\hline
\end{tabular}

Terric Anthrosols (Escalic. Tephric. Technic. Vitric) con relleno de toba cristalítica-lítica (terrazas)

$\begin{array}{lcccccccr}1 & 0-15 & \text { Subangular } & \text { 10YR 6/3 } & 10 \text { YR 4/3 } & 1.4 & 7.5 & 0.95 & \text { Areno francosa } \\ 1 & 15-30 & \text { Subangular y angular } & 10 \text { YR 7/2 } & 10 \text { YR 4/4 } & 1.5 & 7.9 & 1.09 & \text { Areno francosa } \\ & 30-40 & \text { Subangular y angular } & 10 \text { YR 7/2 } & 10 \text { YR 4/3 } & 1.6 & 7.9 & 2.71 & \text { Areno francosa }\end{array}$

Eutric Leptosols/ Eutric Regosols (Tephric. Technic) a partir de toba cristalítica-lítica

$\begin{array}{ccccccccccc}1 & 0-15 & \text { Subangular } & \text { 10YR 7/2 } & 10 \text { YR 3/4 } & 1.6 & 7.7 & 0.33 & \text { Areno francosa } \\ 1 & 0-25 & \text { Subangular } & \text { 10YR 6/2 } & 10 \text { YR 4/3 } & 1.5 & 7.6 & 0.84 & \text { Areno francosa } \\ 1 & 0-20 & \text { Granular } & 10 \text { YR 6/2 } & 10 \text { YR 4/3 } & 1.4 & 7.2 & 1.6 & \text { Franco arcillo arenosa } \\ & 20-30 & \text { Granular y subangular } & 10 \text { YR 6/3 } & 10 \text { YR 4/4 } & 1.4 & 7.3 & 1.36 & \text { Franco arenosa } \\ 8 & 0-15 & \text { Granular y subangular } & 10 \text { YR 2/2 } & 10 \text { YR 4/2 } & 1.5 & * & 0.27 & \text { Franco arenosa } \\ 8 & 0-15 & \text { Prismas. bloques } & \text { 10YR 3/3 } & 10 \text { YR 6/2 } & 1.5 & * & 0.29 & \text { Franca }\end{array}$

Eutric Fluvisols (Technic)

\begin{tabular}{cccccccccc}
5 & $0-10$ & Granular y subangular & 10 YR 4/3 & 10 YR 3/1 & 1.5 & 6.7 & 2.46 & Franco arcillosa \\
5 & $10-40$ & Subangular & 10 YR 4/2 & 10 YR 3/1 & 1.5 & 6.5 & 2.33 & Franco arcillosa \\
& $0-15$ & Granular & 10 YR 4/2 & 10 YR 4/3 & 1.4 & 7.3 & 3.7 & Arcillosa \\
6 & $15-45$ & Subangular & 10 YR 4/2 & 10 YR 4/3 & 1.5 & 7.2 & 2.74 & Arcillosa \\
& $0-15$ & Subangular & 10 YR 3/2 & 10 YR 4/2 & 1.4 & 7 & 3.01 & Franco arcillosa \\
7 & $15-45$ & Subangular & 10YR 3/1 & 10 YR 4/2 & 1.5 & 7.2 & 2.6 & Franco arcillosa \\
7 & $0-30$ & Granular & 10 YR 4/3 & 10 YR 2/1 & 1.4 & 7.5 & 3.15 & Franco arcillosa \\
& $0-30$ & Granular & 10 YR 4/2 & 10 YR 3/3 & 1.4 & 7.6 & 2.46 & Franco arcillosa \\
\hline
\end{tabular}

Dap = densidad aparente; $\mathrm{MO}=$ materia orgánica.

profundidad entre 20 y $30 \mathrm{~cm}$, lo que determina su clasificación taxonómica, y presentan regolita. Los suelos de riolita son negros a pardos oscuros, franco arcillo limosos, presentan agregados granulares y subangulares ( $3 \mathrm{~cm}$ de diámetro), duros a muy duros; ligeramente ácidos y con bajo contenido de $\mathrm{MO}(<2 \%)$.
En el caso de los suelos formados a partir de andesita son pardo rojizos, franco arcillosos, agregados subangulares, duros y bajo contenido de MO (<2\%). Estos suelos ocurren en las crestas y pendientes altas de los lomeríos. b) Eutric Leptosols/Eutric Regosols (Tephic, Technic) o suelo residual. Los suelos se formaron a partir de 
toba cristalina-lítica (Barrio 1); son someros (0-20 o 0-30 cm), sin regolita; pardo grisáceo oscuros a gris pardusco claros, texturas areno francosas y estructura de bloques subangulares. Además, presentan una reacción neutra a moderadamente alcalina. Estos suelos se presentan en la pendiente media de los lomeríos, con fragmentos de artefactos.

c) Terric Anthrosols (Escalic, Technic) o Terrazas. Formados con rellenos de material riolítico (Barrios 1 y 12), de más de $40 \mathrm{~cm}$ de profundidad; pardos oscuros, franco arcillosos a franco limosos, estructuras de bloques subangulares, ligeramente ácidos a alcalinos y contienen entre 1 y $4 \%$ de MO. Los rellenos presentan fragmentos de rocas y fragmentos de artefactos.

d) Terric Anthrosols (Escalic, Technic, Vitric) o Terrazas. Formados por rellenos de toba cristalina-lítica (Barrio 1); de $<50 \mathrm{~cm}$ de profundidad; grises a grises claros en seco, textura arena francosa a franco arenosa y pardos en húmedo, estructura de bloques subangulares, consistencia firme; ligera a moderadamente alcalinos (8.4) y contenidos medios (2 y 3\%) a ricos (4\%) en MO. Los rellenos son homogéneos en granulometría y con fragmentos de artefactos.

e) Eutric Fluvisols (Technic). Los suelos son profundos $(>100 \mathrm{~cm})$ (Barrios 1, 5, 6, y 7); pardos a grises claros, texturas desde gruesas a finas (franco arcillosas o franco arenosas), agregados migajosos a granulares en la capa arable y bloques subangulares en el subsuelo, o bien se pueden encontrar subangulares/granulares, ligeramente firmes a friables. Además, los suelos presentan una reacción ligeramente ácida a ligeramente alcalina, pobres $(<2 \%)$ a medios en MO $(2-3 \%)$ y ocurren en el plano aluvial, con pendientes entre nulas y $<4 \%$.

En el área de influencia del CSMA, la intensa actividad humana modificó a los suelos y formó Anthrosols con calificadores como Escalic que representan a las terrazas o como Technic por el alto contenido de fragmentos de cerámica y restos de obsidiana. La erosión del área ha generado que la profundidad de los suelos se modifique en cortas distancias y los suelos se clasifiquen ya sea como Leptosols o Regosols ( $<$ o $>25 \mathrm{~cm}$ ); además, los suelos son Eutric por el alto porcentaje de bases.

En cuanto a las tobas (tepetates), en los barrios estudiados se encontraron dos tipos (Figura 4): 1) Vítrica (ácida), textura franco arenosa, friable y de colores claros (10YR 6 o 7/1) y polvosa cuando se disgrega; y 2) Cristalina-lítica, de naturaleza ácida, muy dura, colores pardos (10 YR 4/3), e incrustaciones de bombas volcánicas ( $7 \mathrm{~cm}$ de diámetro) de naturaleza riolítica. La toba cristalina-lítica sobrepone a la toba vítrica en el Barrio 1.

\section{Relación entre Tipos de Suelos y Frecuencia de Glifos de Clases de Tierras}

A continuación, se relacionan las clases de tierras (frecuencia de parcelas) con el barrio dónde se representó dominantemente (Cuadro 2) y su posible representación pictórica se ilustra en la Figura 5.

1) Tepetlatlalli se representó en el Barrio 1 (76 parcelas, 27\%). En este barrio ocurren dos tipos de tepetates o tobas, las cuales se ubican en la parte intermedia y baja de la catena y pueden aflorar a la superficie por erosión

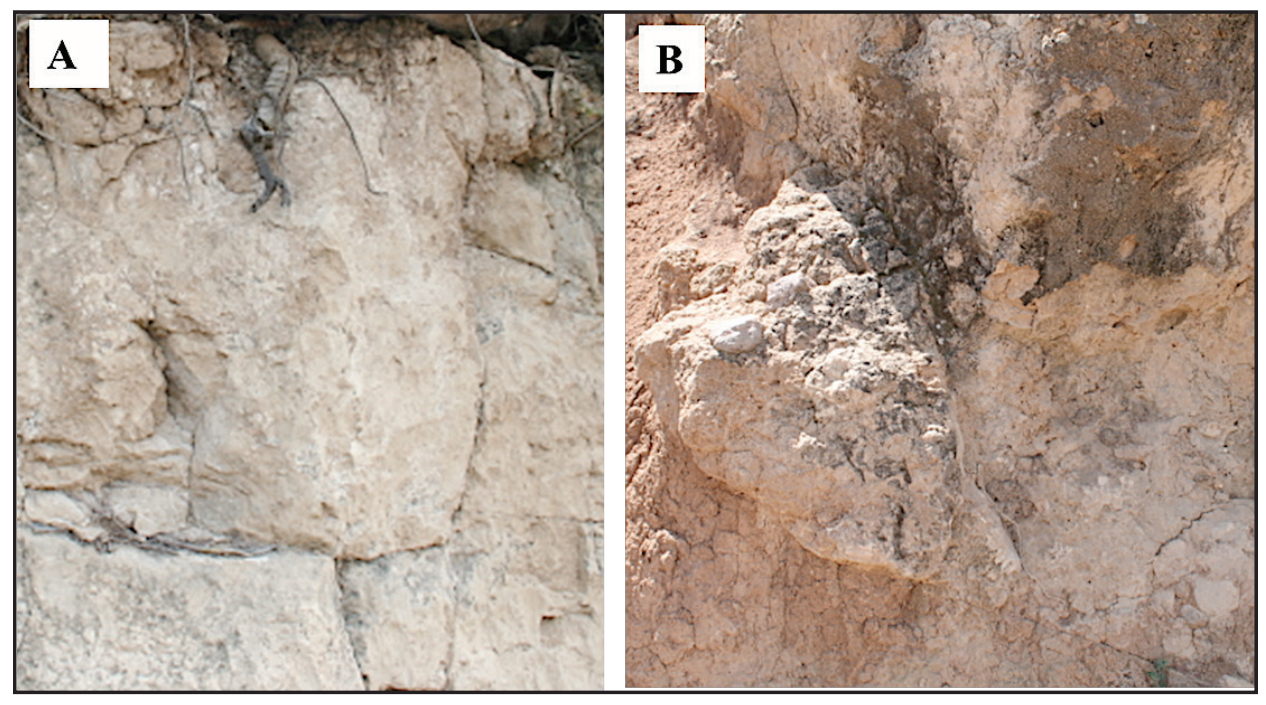

Figura 4. Tobas volcánicas. A) vítrica y B) cristalina-lítica; Barrio 1 de Cuauhtepuztitla. 
Cuadro 2. Número de parcelas (Milcocoli) por clase de tierra en los barrios estudiados.

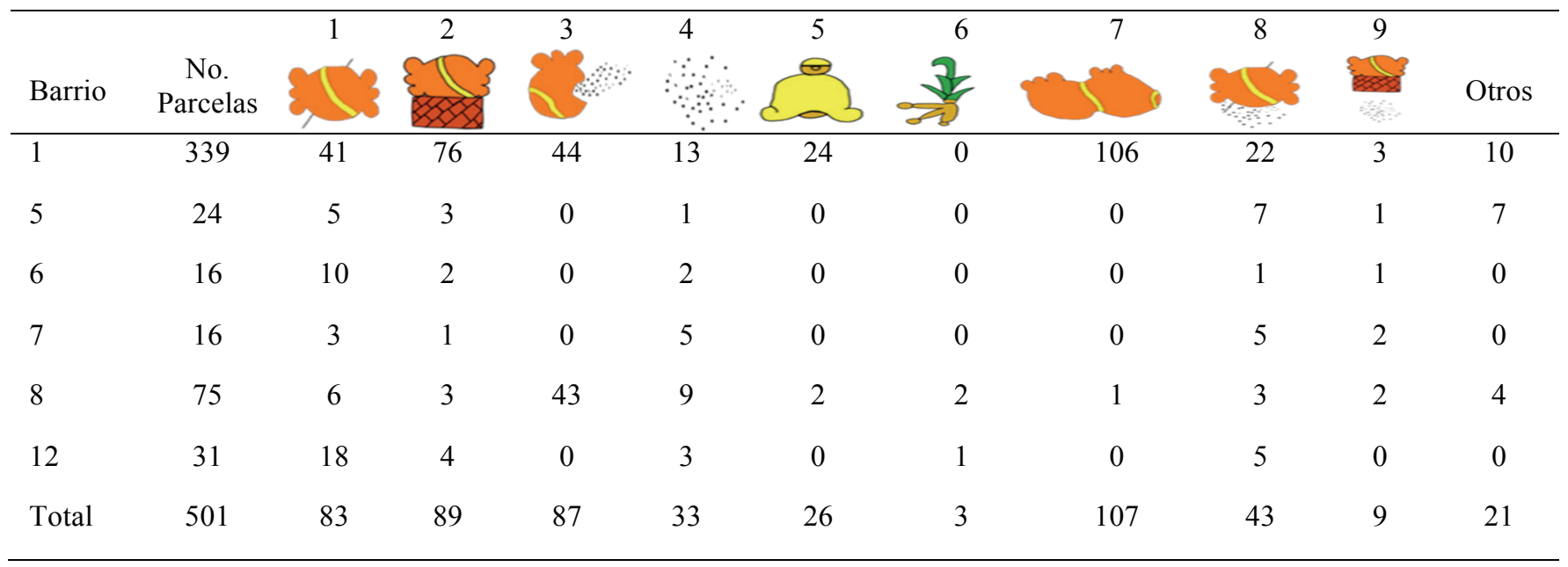

1 Tezoquitl; 2 Tepetlatlalli; 3 Tlalcoxtli; 4 Xalalli; 5 Tlaixtli, 6 Atoctli, 7 Tetlalli, 8 Tezoquitl/Xalalli; 9 Tepetlatlalli/Xalalli.

hídrica o bien formar suelos de diferentes espesores $(<30 \mathrm{~cm}$ de profundidad). También se presentan terrazas y suelos con regolita. En el resto de los barrios, se reporta este glifo entre una y cuatro parcelas.

2) Tetlalli se representó en el Barrio 1 (106 parcelas, $31.2 \%$ ) y en el Barrio 8 (una parcela). Los suelos son someros y con alta pedregosidad conformada de rocas riolíticas subangulares o redondeadas; se localizan en las crestas, terrazas, partes intermedias de los lomeríos, o bien en los suelos reforestados (con eucaliptos, matorrales o malezas). Esta clase de tierra está ausente en el plano aluvial (Barrios 5, 6 y 7).

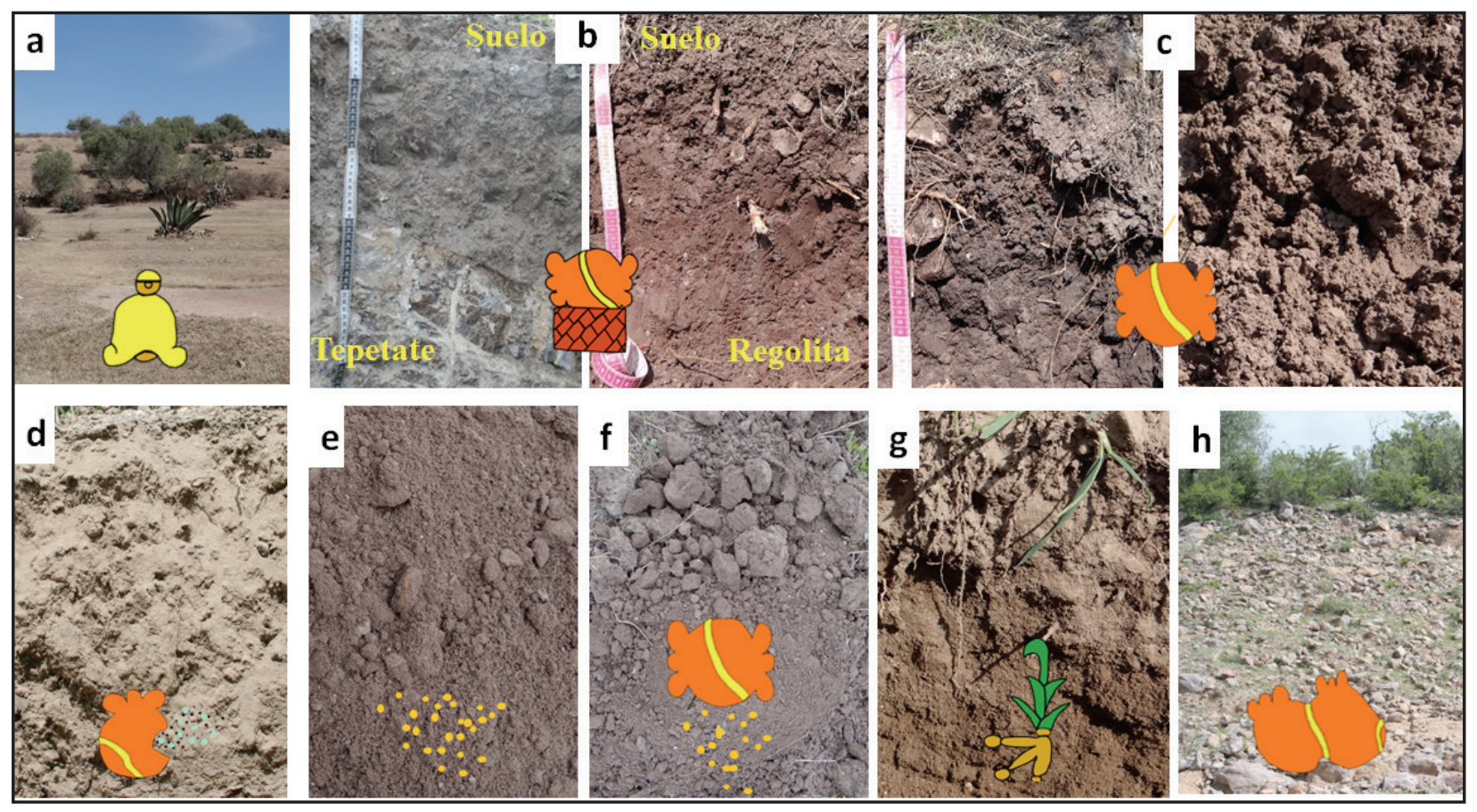

Figura 5. Tipos de suelos de los barrios estudiados y su posible representación pictórica en el CSMA: a) Tlaixtli, suelos de colina o sin uso; b) Tepetlatlalli, suelos de terrazas o de regolita; c) Tezoquitl, tierras con agregados subangulares/ granulares, duros; d) Tlalcoxtli, tierras polvosas; e) tierras con agregados granulares suaves; f) tierras de aluvión pasivo donde se combina Tezoquitl/Xalalli; g) tierras de aluvión activo; h) tierras pedregosas. 
3) Tlalcoztli se localizan en las pendientes bajas, principalmente en el Barrio 8 (43 parcelas, 57\%) y en el Barrio 1 (44 parcelas, 13\%). Los suelos son de colores claros (10 YR 6/1), de consistencia suelta, textura franco arenosa y se formaron a partir de tobas vítricas. Algunas parcelas son agrícolas donde se cultiva avena y maíz, pero con bajo desarrollo (menos de $1 \mathrm{~m}$ de altura), crecen en manchones y con evidencia de clorosis.

4) Xalalli. Son tierras difíciles de establecer debido a que los suelos de texturas franco arenosas o areno francosas fueron comunes en los suelos formados a partir de tobas cristalinas (Barrio 1 con 3.8\% y Barrio 8 con $12 \%$ ), que también reúnen las características de las Tlalcoztlis. En el plano aluvial donde se representan hasta en $31 \%$ (Barrio 5), las texturas son franco arcillosas y dominan agregados granulares $(<3 \mathrm{~mm})$, muy friables.

5) Tezoquitl está representado en el Barrio 12 de manera dominante $(50 \%)$. Este barrio está integrado por lomeríos con suelos residuales negros (riolita) y rojizos (andesita), de textura arcillosa. También se representaron en el Barrio 6, en el plano aluvial (10 parcelas, 62.5\%).

6) Tlaixtli se representó en el Barrio 1 (24 parcelas, $7 \%$ ) y en menor proporción en el Barrio 8 (2 parcelas, 2.7\%). Ambos están geomorfológicamente integrados por crestas, pendientes y lomeríos; no obstante, no se reportó en el Barrio 12 donde también se presentan esas características morfológicas.

7) Tezoquitl/Xalalli se representó en casi todos los barrios en un porcentaje de ocurrencia bajo (1-12\%), pero su mayor porcentaje ocurre en el plano aluvial (77\%).

8) Atoclti está representado por 3 parcelas de los Barrios 8 y 12 (2.6 y 8\% respectivamente). En estos sitios ocurren manantiales durante la época de lluvias y los campesinos colocan abrevaderos.

De acuerdo con Fray Bernardino de Sahagún (1963), los aztecas clasificaban a sus tierras a través de diversos atributos como: textura, agregados, color, contenido orgánico, topografía, génesis, tipos de plantas, fertilidad y prácticas agrícolas. Dada la relevancia de esta clasificación, a continuación, se realiza una discusión de estas propiedades.

Los colores oscuros se encontraron dominantemente en barrios en donde se representaron los Tepetlatlalli, Tezoquitl y Tezoquitl/Xalalli (Barrios 1, 5, 6 y 7); aunque, también ocurren suelos arcillosos pardo rojizos en Barrio 12, en donde dominan los Tezoquitl.
Otro caso son las tierras amarillas, las cuales se han relacionado con los Tlalcoztlis (Williams, 2006), pero los colores claros también se presentan en las terrazas (Anthrosols) y en el plano aluvial (Fluvisols). Estos resultados apuntan que la representación de clases de tierras no está totalmente relacionada con el color, como se hace actualmente por parte de los campesinos (Williams y Ortiz, 1981; Ortiz-Solorio et al., 2005).

En cuanto a la textura, se indica que los acolhuas diferenciaban a las tierras en cinco clases: arcillosa, arenosa y limosa, pedregosa y tepetate (Williams, 2006). En campo en donde se representó al Tezoquitl, los suelos son desde arcillosos hasta franco arcillo arenosos, y no siempre presentaron una consistencia firme, incluso los agregados del plano aluvial son friables (Barrios 5, 6, y 7). Estos resultados contrastan con la interpretación de Williams y Ortiz (1981), quienes indican que se trata de tierras negras y de barro. También es necesario indicar que en el Barrio 12, los agregados presentan una forma peculiar, teniendo como base bloques subangulares con inclusiones de agregados granulares (globular), por lo que el Tezoquitl puede estar representando al tipo de tierra o agregados de consistencia dura (Figura 6a). En lo que respecta a los Xalallis o tierras arenosas no fueron encontradas en los barrios donde aparentemente son dominantes (Barrios 5, 6, y 7). Estos barrios que ocurren en los planos aluviales se presentan texturas franco arcillosas, con agregados granulares de menos de $3 \mathrm{~mm}$ y friables (Figura 6b). Es probable que los habitantes de esta zona hayan clasificado a las partículas del suelo por su tamaño (estructura) y consistencia más que por textura, como se ha indicado por varios autores (Williams y Ortiz-Solorio, 1981; Williams, 2006).

Los Tepetlatlalli o tepetate, Williams (1972) los define como un material que no tenía ni la dureza de una roca ni la de un suelo; además, se indica que es un horizonte cementado que se expone a la superficie por erosión. Por su parte, Williams (1992) reportó que en el CSMA se representaron 27 variantes de tepetates. Esta complejidad de tobas o materiales endurecidos no se encontró en campo, lo que sí se encontró y que reúne esta dureza intermedia son las regolitas ya sea de rocas consolidadas (riolita o andesita) o de tobas (cristalinas). Si la dureza es una propiedad importante dentro de las clasificaciones aztecas, este criterio puede abarcar a las regolitas que reúnen las características de un tepetate (Williams, 1972). 


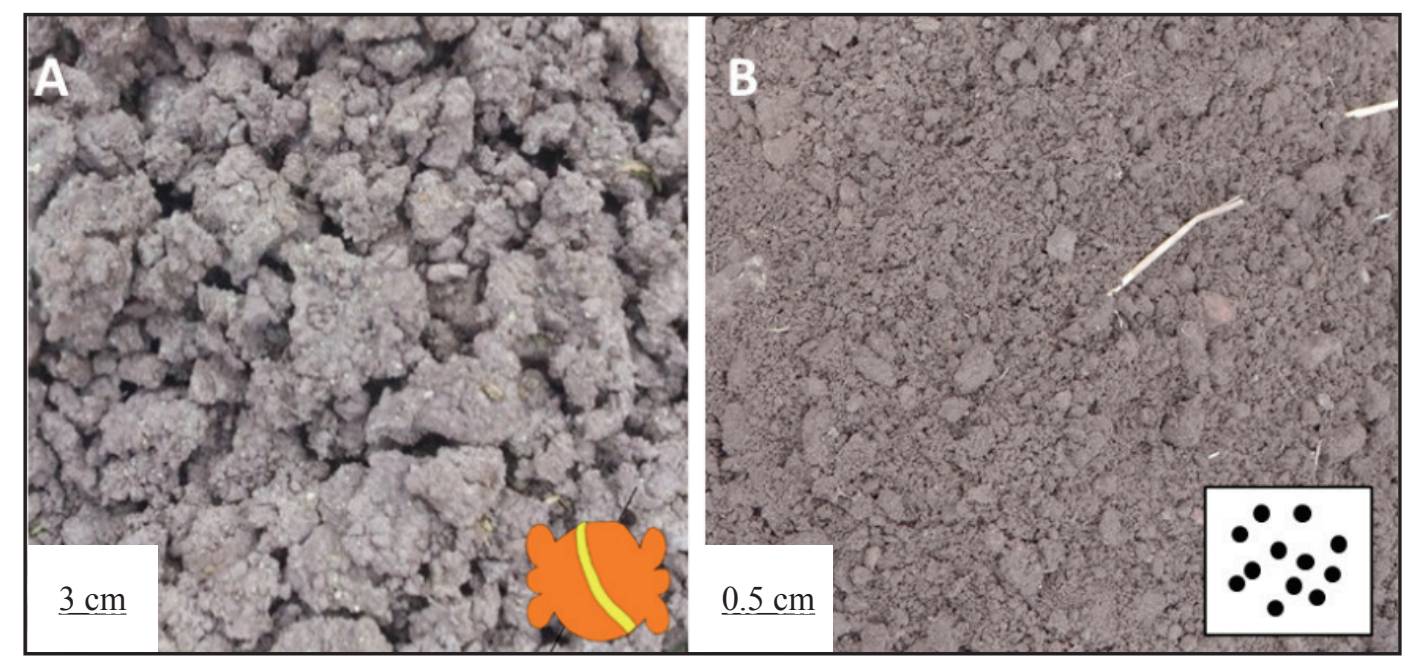

Figura 6. Bloques subangulares y granulares y su posible representación pictográfica. A) Tezoquitl; B) Xalalli.

Otra interpretación del glifo Tepetlatlalli (tepetate), dominantemente en el Barrio 1 (27\%) es que se puede tratar de terrazas (Anthrosols). En el Códice Florentino se reporta una ilustración de cómo los indígenas pulverizaban al tepetate (Fray Bernardino de Sahagún, 1963), como una forma de recuperar a los suelos (Williams y Pierce, 2014). Con esa base, el glifo podría representar la construcción y rellenos de materiales o formación de suelos artificiales.

De acuerdo con Stanchi et al. (2012), las terrazas indican sitios de fuertes asentamientos humanos con alta actividad agrícola, debido a que el relleno que se utiliza para su construcción es un suelo con desarrollo pedogenético limitado, de textura gruesa, pero con alta productividad. Además, las terrazas es una práctica que se origina desde las culturas indígenas como una forma de fertilización, riego y labranza de conservación (Sandor y Eash, 1994; Sandor et al., 1985) ya que reducen la velocidad de escorrentía, aumentan la humedad del suelo y son suelos de excelente calidad agrícola (Sandor et al., 1990; Arnáez et al., 2015).

Los Atoctlis fueron representados solo en los Barrios 8 y 12, donde ocurren actualmente aluviones activos (manantiales y agostaderos). En contraste, los suelos aluviales pasivos (Barrios 5, 6 y 7) pueden estar representados por Tezoquitl/Xalalli o Xalalli; es decir, agregados grandes/ agregados finos y friables, lo que indica que probablemente los acolhuas diferenciaban el origen de las tierras pero también horizontes. Estos resultados contrastan con lo mencionado por Williams (1980) quien indica que la sucesión de capas fue desapercibida por los nahuas.
En el caso de las tierras de Tlalcoztlis, Fray Bernardino de Sahagún (1963), indica que eran tierras arrastradas por el agua y muy fértiles. Incluso, se llegó a calcular que estas tierras podrían producir hasta $1.5 \mathrm{Mg} \mathrm{ha}^{-1}$ de maíz, en años buenos durante el periodo de contacto (Williams, 1989), debido a que son finas, amarillas y muy fértiles (Williams, 2006). En la presente investigación, las Tlalcoztlis son de baja calidad agrícola, amarillas pero de texturas gruesas (arenas finas), de consistencia polvosa y se formaron de tobas vítricas (tepetates).

Finalmente, el glifo Tetlalli representa suelos pedregosos los cuales son muy comunes en el Barrio 1. Estos suelos son el resultado de la erosión de las tobas cristalíticas-líticas, donde el material fino es removido y las bombas volcánicas (redondas) se quedan en la superficie o bien de rocas subangulares comunes en los Leptosols/Regosols formados a partir de riolita. El origen coluvial o eólico pueden ser una explicación de las variantes de este glifo.

La fertilidad es otro aspecto importante. Las tierras de mayor calidad agrícola por sus propiedades edáficas se localizaron en las terrazas y en el plano aluvial $(40 \mathrm{~cm}$ o más de profundidad, friables y alto contenido de $\mathrm{MO}$ hasta 4\%). En el plano aluvial (Barrios 5, 6, y 7) no está representado los Atoctlis, sino Tezoquitl, Tezoquitl/ Xalalli y Xalalli. De acuerdo con los productores, en estas tierras se pueden alcanzar hasta $3 \mathrm{Mg} \mathrm{ha}^{-1}$, sin fertilizantes y bajo secano.

En la Figura 7 se muestra el cultivo de maíz con el fin de ilustrar el vigor, densidad de plantas y deficiencias nutrimentales en diferentes clases de tierras. 


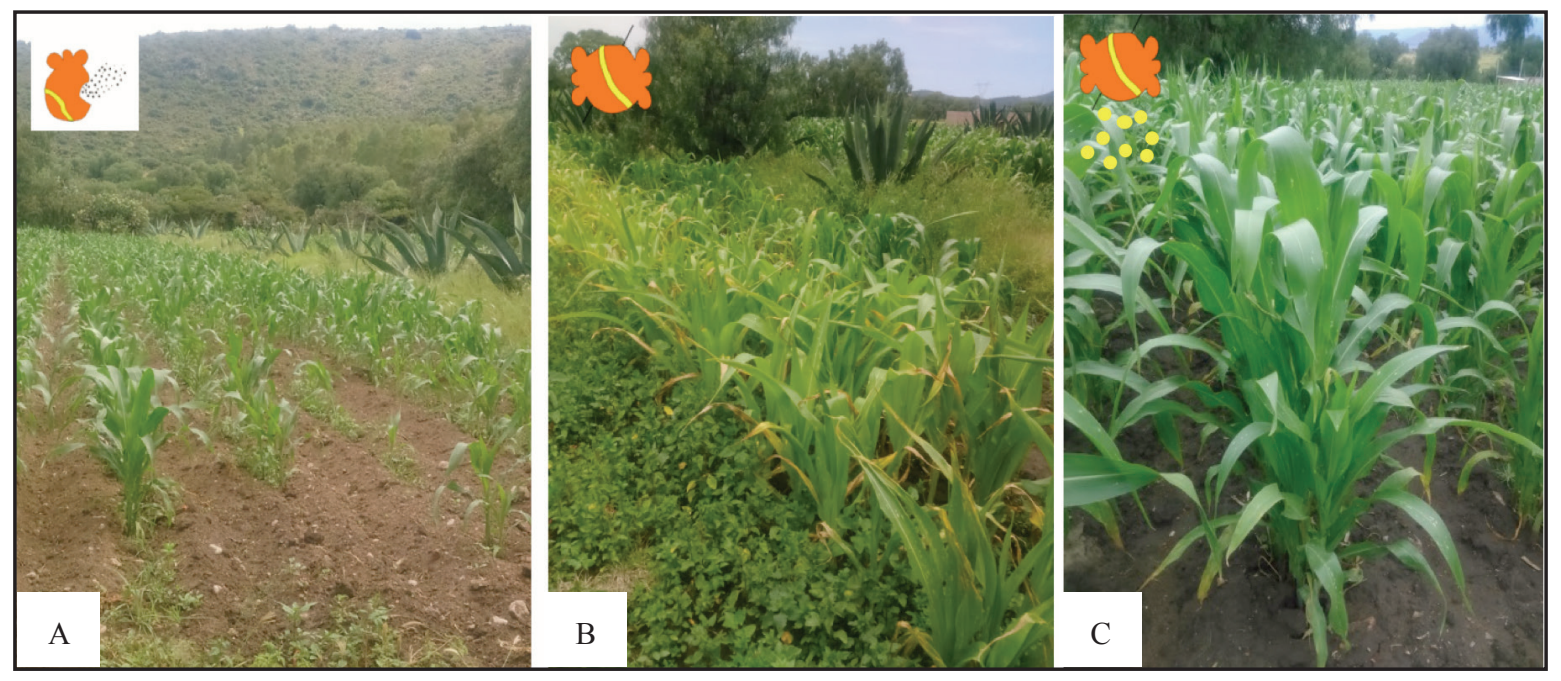

Figura 7. A) Tierra de Tlalcoztli (Regosols); B) Tezoquitl (Regosols); C) Tezoquitl/Xalalli (Fluvisols) en cultivo de maíz.

\section{CONCLUSIONES}

- En los cinco barrios estudiados del área de influencia del Códice de Santa María Asunción (CSMA) ocurren cuatro tipos de suelos Leptosols/ Regosols (a partir de riolitas, andesitas y tobas), Anthrosols y Fluvisols, los cuales se distribuyen en catenas (crestas, pendientes y planos aluviales). Los materiales parentales que se presenta en la zona son tobas (vítricas y cristalinas), rocas consolidadas (riolitas y andesitas) y sedimentos aluviales.

- Los nahuas clasificaron a las tierras con base en el tamaño de los agregados, consistencia y origen. Los Tepetlatlali pueden representar a los Anthrosols (terrazas) y a las regolitas de los materiales parentales; los Tezoquitl a las tierras o a los bloques subangulares/ granulares, duros de $>3 \mathrm{~cm}$; las Tlalcoztlis a las tierras polvosas, de arenas finas, que se formaron de tobas vítricas. Los suelos aluviales activos los representaron como Atoctlis, y los suelos aluviales pasivos como Tezoquitl/Xalalli o Xalallis. Las tierras más fértiles son las que ocurren en el plano aluvial y las terrazas; mientras que las tierras de menor calidad agrícola son las Tlalcoztlis. En el área de influencia del CSMA no ocurre una amplia variedad de tipos de suelos por lo que las variantes en los Tepetlatlalis o Tlalcoztlis deben de ser analizados todavía con mayor detalle para su interpretación.

\section{LITERATURA CITADA}

Arnáez, J., N. Lana-Renault, T. Lasanta, P. Ruiz-Flaño, and J. Castroviejo. 2015. Effects of farming terraces on hydrological and geomorphological processes. A review. Catena 128: 122-134.

Cachón, H. E., H. Nery y H. E. Cuanalo de la Cerda. 1976. Los suelos del área de influencia de Chapingo. Rama de suelos sección de Pedología. Colegio de Postgraduados. Chapingo, México.

Códice de Santa María Asunción y el apeo y deslinde de tierras (de los terrenos) de Santa María de la Asunción. MS de Olagüíbel. 1497 bis. Biblioteca Nacional de México. México, D. F.

García, E. 1973. Modificaciones al sistema de clasificación climática de Köppen: Para adaptarlo a las condiciones de la República Mexicana. Instituto de Geografía UNAM. México, D. F.

GEM (Gobierno del Estado de México). 1993. Atlas general de Estado de México, región Tepetlaoxtoc. Secretaria de Finanzas y Planeación. Estado de México. México.

Harvey, H. R. and B. J. Williams. 1980. Aztec arithmetic: Positional notation and area calculation. Science 210: 499-505.

Ingersoll, R. V., T. F. Bullard, R. L. Ford, J. P. Grimm, J. D. Pickle, and S. W. Sares. 1984. The effect of grain size on detrital modes: a test of Gazzi-Dickinson point-counting method. J. Sediment. Petrol. 54: 103-116.

IUSS Working Group WRB (International Union of Soil SciencesWorld Reference Base for Soil Resources). 2015. Base referencial mundial del recurso suelo 2014, Actualización 2015. Sistema internacional de clasificación de suelos para la nomenclatura de suelos y la creación de leyendas de mapas de suelos. Informes sobre recursos mundiales de suelos 106 . FAO, Roma

López-Corral, A. 2011. Los glifos de suelo en códices Acolhua de la colonia temprana: Un reanálisis de su significado. Desacatos 37: 145-62. 
Ortiz-Solorio, C. A., Ma. del C. Gutiérrez-Castorena, A. L. Licona-Vargas, and P. Sánchez-Guzmán. 2005. Contemporary influence of indigenous soil (land) classification in Mexico. Eurasian Soil Sci. 38: s89-s94.

Sahagún, Bernardino de. 1963. Florentine Codex: General history of the things of the New Spain. The School of American Research. University of Utah Press. Salt Lake City, UT, USA.

Sandor, J. A. and N. S. Eash. 1994. Ancient agricultural soils in the Andes of Southern Peru. Soil Sci. Soc. Am. J. 59: 170-179.

Sandor, J. A., P. L. Gersper, and J. W. Hawley. 1985. Soils at prehistoric agricultural terracing sites in New Mexico: I. Site placement, soil morphology, and classification. Soil Sci. Soc. Am. J. 50: 166-173.

Sandor, J. A., P. L. Gersper, and J. W. Hawley. 1990. Prehistoric agricultural terraces and soils in Mimbres area, New México. World Archaeol. 22: 70-86.

Stanchi, S., M. Freppaz, A. Agnelli, T. Reinsch, and E. Zanini. 2012. Properties, best management practices and conservation of terraced soils in Southern Europe (from Mediterranean areas to the Alps): A review. Quat. Int. 265: 90-100.

Van Reeuwijk, R. 2003. Procedimientos para el análisis de suelos clasificación y correlación. Colegio de Postgraduados. Montecillo, Texcoco, México.

Williams, B. J. 1972. Tepetate in the Valley of Mexico. Ann. Assoc. Am. Geog. 62: 618-626.

Williams, B. J. 1980. Pictorical representation of soils in the Valley of Mexico: Evidence from the codex Vergara. Geosci. Man 21: 51-62.

Williams, B. J. 1981. La clasificación náhua de suelos. pp. 233-236. In: T. Rojas Rábiela y W. T. Sanders (eds.). 1981. Historia de la agricultura: Época prehispánica-Siglo XVI. INAH. México, D. F.
Williams, B. J. 1989. Contact period rural overpopulation in the basin of Mexico: Carrying-capacity models tested with documentary data. Am. Antiq. 54: 715-732.

Williams, B. J. 1991. The lands and political organization of a rural Tlaxilacalli in Tepetlaoztoc, c A.D. 1540. pp. 187-208. In: H. R. Harvey (ed.). Land and politics in the Valley of Mexico: A two thousand year perspective. University of New Mexico Press. Albuquerque, NM, USA.

Williams B. J. 1992. Tepetate in Sixteen Century and contemporary folk terminology Valley of Mexico. Terra 10: 483-493.

Williams, B. J. 2006. Aztec soil knowledge: Classes, management, and ecology. pp. 17-41. In: B. P. Warkentin (ed.). Footprints in the soil: People and ideas in the soil history. Elsevier. Amsterdam, The Netherlands.

Williams, B. J. and C. A. Ortiz-Solorio. 1981. Middle american folk soil taxonomy. Ann. Assoc. Am. Geog. 71: 335-358.

Williams, B. J. and H. R. Harvey. 1988. Content, provenience, and significance of the Codex Vergara and the Codice de Santa Maria Asuncion. Am. Antiq. 53: 337-351.

Williams, B. J. and H. R. Harvey. 1997. The codice de Santa Maria Asunción: Facsimile and commentary: Households and lands in sixteenth-century Tepetlaoztoc. University of Utah Press. Salt Lake City, UT, USA.

Williams, B. J. and K. J. Pierce. 2014. Evidence of Acolhua science and pictorial land records. pp. 147-164. In: J. Lee and G. Brokaw (eds.). Texcoco: Prehispanic and colonial perspectives. University Press of Colorado. Boulder, CO, USA.

Williams, B. J. and M. C. Jorge y Jorge. 2008. Aztec arithmetic revisited: Land area algorithms and Acolhua congruence arithmetic. Science 320: 72-77.

Williams, H., F. J. Turner y G. C. M. 1968. Petrografía: Introducción al estudio de la rocas en secciones delgadas. C.E.C.S.A. México, D. F. 